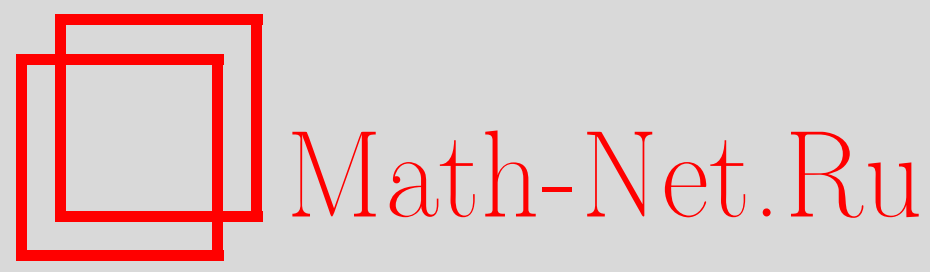

О. В. Бесов, Пространства функций дробной гладкости на нерегулярной области, Матем. заметки, 2003, том 74, выпуск 2, 163-183

DOI: https://doi.org/10.4213/mzm253

Использование Общероссийского математического портала Math-Net.Ru подразумевает, что вы прочитали и согласны с пользовательским соглашением http://www . mathnet.ru/rus/agreement

Параметры загрузки:

IP : 54.209 .52 .79

26 апреля 2023 г., 15:39:22

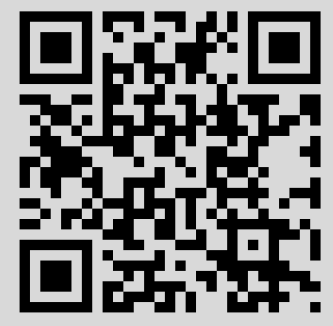




\section{ПРОСТРАНСТВА ФУНКЦИЙ ДРОБНОЙ ГЛАДКОСТИ НА НЕРЕГУЛЯРНОЙ ОБЛАСТИ}

\section{O. В. Бесов}

В статье изучаются пространства $B_{p q}^{s}(G), L_{p q}^{s}(G)$ функций с положительным показателем гладкости $s>0$, заданных на области $G \subset \mathbb{R}^{n}$. Для области $G$ с определенными геометрическими свойствами установлено вложение $B_{p p}^{s}(G)=L_{p p}^{s}(G) \subset L_{q}(G)$, $1<p<q<\infty$, при соотношении параметров, определяемом этими геометрическими свойствами.

Библиография: 20 названий.

В статье изучаются пространства $B_{p q}^{s}(G), L_{p q}^{s}(G)$ функций $f$ с положительным показателем гладкости $s>0$, заданных на области $G \subset \mathbb{R}^{n}$. Нормы в этих пространствах определены с помощью интегральных норм разности функции $f$ порядка $m>s$, pacсматриваемой как функция точки области и шага разности.

Для произвольной области $G \subset \mathbb{R}^{n}$ возможна характеризация этих пространств в терминах локальных апшроксимаций функции многочленами степени $m-1$.

Для области $G$ с определенными геометрическими свойствами устанавливается вложение

$$
B_{p p}^{s}(G)=L_{p p}^{s}(G) \subset L_{q}(G), \quad 1<p<q<\infty,
$$

в лебегово пространство $L_{q}(G)$ при соотношении параметров $s, p, q, n$, определяемом этими геометрическими свойствами. Это соотношение особенно просто выглядит для области с $\sigma$-условием Джона ( $\sigma$-John domain), $\sigma \geqslant 1$. Такая область при $\sigma>1$ может в окрестности точки границы иметь вид пика, направленного во внешность области.

Вложение $W_{p}^{s}(G) \subset L_{q}(G)$ пространств Соболева $(s \in \mathbb{N})$ для области с нерегулярной границей изучалось в ряде работ (см. [1], [2]). Отметим, что здесь, как и в [1], [2], решающую роль при доказательстве вложения играет надлежащим образом построенное интегральное представление функции по "гибкому конусу". В данном случае это интегральное представление функции через ее локальные уклонения от многочленов, построение которого приводится.

Пространства $B_{p q}^{s}(G), L_{p q}^{s}(G)$ на области $G$ с регулярной (т.е. локально липшицевой) границей изучены достаточно полно. Установлено, в частности, что нормы, построенные с помощю разностей различных порядков $m^{\prime}, m^{\prime \prime}$, эквивалентны друг другу. Здесь будет показано, что на области с нерегулярной границей такой эквивалентности, вообще говоря, нет.

Работа выполнена при финансовой поддержке Российского фонда фундаментальных исследований, проект 02-01-00602, программы "Ведушие научные школы", проект 00-15-96047, и фонда INTAS, грант 99-01080. 
1. Обозначения и определения. Пусть $\mathbb{N}$-множество натуральных чисел, $n \in \mathbb{N}$, $n \geqslant 2, \mathbb{R}^{n}$ - евклидово пространство точек $x=\left(x_{1}, \ldots, x_{n}\right)=\sum_{1}^{n} x_{i} e^{i}, e^{i}$-орты стандартного базиса. Для $x, y \in \mathbb{R}^{n}, t>0, E \subset \mathbb{R}^{n}$ пусть $[x, y]$-отрезок с концами в точках $x, y ; y+t E=\{x: x=y+t z, z \in E\}$,

$$
B(x, t)=\{y:|y-x| \leqslant t\}=x+B(0, t), \quad B_{0}=B(0,1),
$$

$\chi$ - характеристическая функция шара $B_{0}$ или отрезка $[-1,1]$.

При $m \in \mathbb{N}$ положим

$$
\begin{aligned}
\Delta^{m}(y) f(x) & =\sum_{j=0}^{m}(-1)^{m-j}\left(\begin{array}{c}
m \\
j
\end{array}\right) f(x+j y), \\
\Delta^{m}(y, E) f(x) & = \begin{cases}\Delta^{m}(y) f(x) & \text { при } B(x, m|y|) \subset E, \\
0 & \text { при } B(x, m|y|) \not \subset E,\end{cases}
\end{aligned}
$$

при $t>0$

$$
\delta^{m}(t, E) f(x)= \begin{cases}\left|B_{0}\right|^{-1} \int_{|y|<1}\left|\Delta^{m}(t y) f(x)\right| d y & \text { при } B(x, m t) \subset E, \\ 0 & \text { при } B(x, m t) \not \subset E .\end{cases}
$$

Все встречающиеся множества измеримы по Лебегу, $|E|$ - мера Лебега множества $E \subset \mathbb{R}^{n}$. Все встречающиеся функции действительнозначны и локально суммируемы.

Для $f: E \rightarrow \mathbb{R}, 1 \leqslant p \leqslant \infty$ положим

$$
\|f\|_{p, E}=\left\|f \mid L_{p}(E)\right\|=\left(\int_{E}|f(x)|^{p} d x\right)^{1 / p}, \quad\|f\|_{p}=\|f\|_{p, \mathbb{R}^{n}}
$$

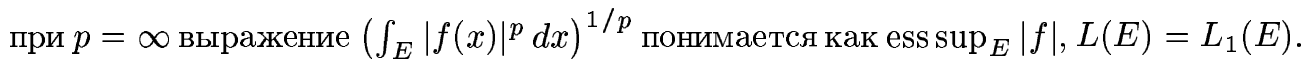

При $1<q<\infty$ через $L_{q}^{*}$ обозначается пространство измеримых на $(0,1)$ функций $g$ с нормой

$$
\left\|g \mid L_{q}^{*}\right\|=\left(\int_{0}^{1}|g(t)|^{q} \frac{d t}{t}\right)^{1 / q}<\infty, \quad L_{\infty}^{*}=L_{\infty}((0,1)) .
$$

Через $G$ будем обозначать область в $\mathbb{R}^{n}$. При $\delta>0$ полагаем $G_{\delta}=\{x: x \in G$, $\operatorname{dist}(x, \partial G)>\delta\}$. При этом $\delta$ считаем столь мальм, что $G_{\delta} \neq \varnothing$.

ОПреДЕЛЕНИЕ 1.1. Символом $B_{p q, r}^{s(m)}(G)$ при $1 \leqslant p, q, r \leqslant \infty, 0<s<m, m \in \mathbb{N}$ будем обозначать банахово пространство функций $f: G \rightarrow \mathbb{R}^{1}$ с нормой

$$
\left\|f\left|B_{p q, r}^{s(m)}(G)\|=\| f\right| L_{r}\left(G_{\delta}\right)\right\|+\left\|f \mid b_{p q}^{s(m)}(G)\right\|,
$$

где

$$
\left\|f \mid b_{p q}^{s(m)}(G)\right\|=\left(\int_{m|h|<1}\left(\frac{\left\|\Delta^{m}(h, G) f\right\|_{p}}{|h|^{s}}\right)^{q} \frac{d h}{|h|^{n}}\right)^{1 / q}
$$


ОПредЕЛЕНИЕ 1.2. Символом $L_{p q, r}^{s(m)}(G)$ при $1<p, q \leqslant \infty, 0<s<m, m \in \mathbb{N}$ будем обозначать банахово пространство функций $f: G \rightarrow \mathbb{R}^{1}$ с нормой

$$
\left\|f\left|L_{p q, r}^{s(m)}(G)\|=\| f\right| L_{r}\left(G_{\delta}\right)\right\|+\left\|f \mid l_{p q}^{s(m)}(G)\right\|
$$

где

$$
\left\|f \mid l_{p q}^{s(m)}(G)\right\|=\left\|\left(\int_{0}^{1 / m}\left(\frac{\delta^{m}(t, G) f}{t^{s}}\right)^{q} \frac{d t}{t}\right)^{1 / q}\right\|_{p} .
$$

При $r=p$ вместо $B_{p q, r}^{s(m)}(G), L_{p q, r}^{s(m)}(G)$ и т.п. будем писать $B_{p q}^{s(m)}(G), L_{p q}^{s(m)}(G)$ и т.п. При $m-1 \leqslant s<m$ верхний индекс $(m)$ в обозначениях пространств будем опускать.

Пространства $B_{p q}^{s}\left(\mathbb{R}^{n}\right), L_{p q}^{s}\left(\mathbb{R}^{n}\right)\left(=F_{p q}^{s}\left(\mathbb{R}^{n}\right)\right)$ изучены достаточно подробно (см. [3]-[5]). Характеризация пространств Лизоркина-Трибеля $L_{p q}^{s}$ на $\mathbb{R}^{n}$ и на областях $G \subset \mathbb{R}^{n}$ с достаточно гладкой границей в терминах шаровых средних от абсолютных величин разностей была предложена Стрихартцем [6] при $q=2$ (пространства бесселевых потенциалов) и в общем случае Лизоркиным [7]. Другие характеризации пространств $B_{p q}^{s}(G), L_{p q}^{s}(G)$ на области $G$ с регулярной границей можно найти в [5], [8].

Введем в рассмотрение также пространства функций, характеризуемых определенными интегральными оценками для их локальных приближений алгебраическими многочленами. Примем некоторые обозначения:

$$
\tau_{a} f(x)=f(x+a), \quad a \in \mathbb{R}^{n}, \quad \sigma_{t} f(x)=f(t x), \quad t>0,
$$

$\mathscr{P}_{m-1}$ - подпространство пространства $L\left(B_{0}\right)$, состоящее из всех многочленов вида $\sum_{|\alpha| \leqslant m-1} c_{\alpha} x^{\alpha}$ (степени не вьше $m-1$ ),

$$
\begin{gathered}
\pi=\pi^{(m-1)} \quad \text { - некоторьй проектор } L\left(B_{0}\right) \text { на } \mathscr{P}_{m-1}, \\
\pi_{a, t}=\tau_{a}^{-1} \circ \sigma_{t}^{-1} \circ \pi \circ \sigma_{t} \circ \tau_{a}, \\
D_{m-1}(t, E) f(x)=D_{m-1}(t, B(x, t) \cap E) f(x) \\
= \begin{cases}t^{-n}\left\|f-\pi_{x, t} f \mid L(B(x, t))\right\| & \text { при } B(x, t) \subset E, \\
0 & \text { при } B(x, t) \not \subset E .\end{cases}
\end{gathered}
$$

ЗАмЕЧАниЕ 1.1 [9]. Функционалы $D_{m-1}(t, G) f$, образованные с помощью двух разных проекторов $\pi$ и $\widehat{\pi}$, эквивалентны с постоянньпи, не зависящими от $f, x, t$. В самом деле,

$$
\begin{aligned}
\left\|f-\widehat{\pi}_{x, t} f \mid L(B(x, t))\right\| & =\left\|f-\pi_{x, t} f-\widehat{\pi}_{x, t}\left(f-\pi_{x, t} f\right) \mid L(B(x, t))\right\| \\
& \leqslant(1+\|\widehat{\pi}\|)\left\|f-\pi_{x, t} f \mid L(B(x, t))\right\| .
\end{aligned}
$$

Из аналогичных оценок ясно также, что $D_{m-1}(t, G) f$ останется эквивалентным после замены в нем приближения функции с помощью проекционного многочлена на ее наилучшее приближение. 
ОПРЕДЕЛЕНИЕ 1.3. При $1 \leqslant p, q, r \leqslant \infty, 0<s<m, m \in \mathbb{N}$ обозначим через $\widetilde{B}_{p q, r}^{s(m)}(G), \widetilde{L}_{p q, r}^{s(m)}(G)$ пространства функций $f: G \rightarrow \mathbb{R}^{1}$ с конечными нормами соответственно

$$
\begin{aligned}
\left\|f \mid \widetilde{B}_{p q, r}^{s(m)}(G)\right\| & =\left\|f\left|L_{r}\left(G_{\delta}\right)\|+\| f\right| \widetilde{b}_{p q}^{s(m)}(G)\right\| \\
\left\|f \mid \widetilde{L}_{p q, r}^{s(m)}(G)\right\| & =\left\|f\left|L_{r}\left(G_{\delta}\right)\|+\| f\right| \widetilde{l}_{p q}^{s(m)}(G)\right\|
\end{aligned}
$$

где

$$
\begin{aligned}
& \left\|f \mid \widetilde{b}_{p q}^{s(m)}(G)\right\|=\left(\int_{0}^{1}\left\|D_{m-1}(t, G) f\right\|_{p}^{q} t^{-s q} \frac{d t}{t}\right)^{1 / q}, \\
& \left\|f \mid \tilde{l}_{p q}^{s(m)}(G)\right\|=\left\|\left(\int_{0}^{1}\left(D_{m-1}(t, G) f\right)^{q} t^{-s q} \frac{d t}{t}\right)^{1 / q}\right\|_{p}
\end{aligned}
$$

Через $b_{p q}^{s(m)}(G), l_{p q}^{s(m)}(G), \widetilde{b}_{p q}^{s(m)}(G), \widetilde{l}_{p q}^{s(m)}(G)$ будем обозначать полунормированные пространства с полунормами, введенными в определениях 1.1-1.3.

Пространства функций, характеризуемых локальными приближениями многочленами, изучались во многих работах, см., например, [4], [9]-[11]. Ближайшими к представленным здесь результатам о совпадении $B_{p q}^{s}(G)=\widetilde{B}_{p q}^{s}(G), L_{p q}^{s}(G)=\widetilde{L}_{p q}^{s}(G)$ являются результаты Аджиева [9], установившего их для областей $G$ с регулярной границей. Этим он дополнил для пространств $B_{p q}^{s}(G)$ предшествующие результаты Ильина (см. [4]) и распространил на области более общего вида результаты Трибеля [10] для областей с бесконечно гладкой границей. Отметим еще результаты Девора и Шарпли [12], исследовавших для регулярной области $G \subset \mathbb{R}^{n}$ шкалу пространств $C_{p}^{s}(G)=\widetilde{L}_{p, \infty}^{s}(G)$. Другие справки исторического характера можно найти в [4], [5], [8], [9], [11].

Из результатов, относящихся к произвольной области $G \subset \mathbb{R}^{n}$, отметим результат Кальдерона [13] о характеризации пространства Соболева $W_{p}^{l}(G)$ в терминах локальных аппроксимаций.

2. Эквивалентные нормировки пространств $b_{p q}^{s(m)}(G), l_{p q}^{s(m)}(G)$. В [14] установлены следуюшие две теоремы.

ТЕОрема 2.1. Для произвольной области $G \subset \mathbb{R}^{n} n p u 0<s<m, 1 \leqslant p, q \leqslant \infty$ полунормы $\left\|f\left|b_{p q}^{s(m)}(G)\|u\| f\right| \widetilde{b}_{p q}^{s(m)}(G)\right\|$ әквивалентны.

ТЕОРема 2.2. Для произвольной области $G \subset \mathbb{R}^{n}$ при $0<s<m$ и при $1<p$, $q<\infty$ или $1<p \leqslant q=\infty$ полунормы $\left\|f\left|l_{p q}^{s(m)}(G)\|u\| f\right| \widetilde{l}_{p q}^{s(m)}(G)\right\|$ әквивалентны.

3. Интегральное представление функций. Построим некоторое интегральное представление функции $f \in L\left(\mathbb{R}^{n}, \mathrm{loc}\right)$, которое будет использовано при доказательстве теоремы вложения в п. 4.

Пусть $m \in \mathbb{N}$,

$$
\eta \in C_{0}^{\infty}\left(\mathbb{R}^{n}\right), \quad \operatorname{supp} \eta \in B\left(0, \frac{1}{m}\right), \quad \int \eta(x) d x=1 .
$$

Пусть $T>0, c_{0}>0$, функции

$$
\tau:(0, T) \rightarrow \mathbb{R}^{n}, \quad r:(0, T) \rightarrow(0, \infty)
$$


непрерьвны и кусочно непрерьвно дифференцируемы,

$$
\begin{gathered}
\tau(t) \rightarrow 0, \quad r(t) \rightarrow 0 \quad \text { при } t \rightarrow 0, \\
\left|\tau^{\prime}(t)\right| \leqslant 1, \quad\left|r^{\prime}(t)\right| \leqslant c_{0} \quad \text { при } 0<t \leqslant T, \quad r_{1}(t)=\frac{r(t)}{t} .
\end{gathered}
$$

Рассмотрим функцию $g \in L\left(\mathbb{R}^{n}, \mathrm{loc}\right)$, и пусть 0 - ее точка Лебега. Введем усреднение

$$
g_{t}(0)=\int \sum_{j=1}^{m} \sum_{k=0}^{m-1} a_{j} c_{k}\left(r_{1}(t)\right) g\left(j r(t) v+\tau(t)\left(1+k r_{1}(t)\right)\right) \eta(v) d v
$$

где

$$
a_{j}=(-1)^{j-1}\left(\begin{array}{c}
m \\
j
\end{array}\right)
$$

так что

$$
\sum_{j=1}^{m} a_{j}=1, \quad \sum_{j=1}^{m} j^{l} a_{j}=-\left.\left(u \frac{d}{d u}\right)^{l}(1-u)^{m}\right|_{u=1}=0 \quad \text { при } l=1, \ldots, m-1,
$$

a $\left\{c_{k}(t)\right\}_{k=0}^{m-1}$ является решением системы $m$ линейных уравнений

$$
\sum_{k=0}^{m-1} c_{k}(t)=1, \quad \sum_{k=0}^{m-1} c_{k}(t)(1+k t)^{l}=0, \quad l=1,2, \ldots, m-1 .
$$

Определитель $D(t), t>0$, системы (3.3) является определителем Вандермонда, так что при $t>0$ система (3.3) имеет единственное решение. По известной формуле вычисления определителя Вандермонда

$$
D(t)=b t^{(m+1)(m-2) / 2} .
$$

Решение системы (3.3) можно найти по правилу Крамера. Пусть определитель $D_{k}(t)$ отличается от $D(t)$ заменой в последнем $k$-го столбца на столбец правых частей системы (3.3), лишш первьй элемент которого отличен от нуля. Раскладывая $D_{k}(t)$ по элементам первой строки, получим $D_{k}(t)=(-1)^{k-1} D_{k}^{(m-1)}(t)$, где $D_{k}^{(m-1)}(t)$ - определитель порядка $m-1$. После вынесения из каждого его столбца множителя, совпадающего с первым элементом этого столбца, он становится определителем Вандермонда порядка $m-1$. Поэтому

$$
D_{k}(t)=Q_{m-1, k}(t) t^{m(m-3) / 2},
$$

где $Q_{m-1, k}-$ многочлен степени $m-1$. Отсюда

$$
c_{k}(t)=Q_{m-1, k}(t) t^{-m+1} .
$$

Покажем, что для произвольного многочлена $P_{m-1}(x)=\sum_{|\alpha| \leqslant m-1} c_{\alpha} x^{\alpha}$ с постоянными коэффициентами введенное усреднение $\left(P_{m-1}\right)_{t}(0)$ не зависит от $t>0$. Достаточно рассмотреть лишь случай $P_{m-1}(x)=p_{\alpha}(x)=x^{\alpha}, 1 \leqslant|\alpha| \leqslant m-1$. Но

$$
\begin{aligned}
{\left[j r(t) v+\tau(t)\left(1+k r_{1}(t)\right)\right]^{\alpha} } & =\sum_{\beta \leqslant \alpha} c_{\alpha \beta}(j r(t) v)^{\beta}\left[\tau(t)\left(1+k r_{1}(t)\right)\right]^{\alpha-\beta} \\
& =\sum_{\beta \leqslant \alpha} c_{\alpha \beta}(j r(t))^{|\beta|} v^{\beta}\left(1+k r_{1}(t)\right)^{|\alpha-\beta|} \tau(t)^{\alpha-\beta},
\end{aligned}
$$

где $\beta \leqslant \alpha$ означает, что $\beta_{i} \leqslant \alpha_{i}$ при $i=1,2, \ldots, n$. 
Тогда в силу соотношений ортогональности из (3.2), (3.3) имеем

$$
\left(p_{\alpha}\right)_{t}(0)=0 \quad \text { при }|\alpha| \geqslant 1 \text {. }
$$

В силу же первых соотношений из (3.2), (3.3)

$$
\left(p_{0}\right)_{t}(0)=\int \eta(v) d v=1=p_{0}(0),
$$

что и требовалось доказать. Отсюда

$$
\frac{\partial}{\partial t} g_{t}(0)=\frac{\partial}{\partial t}\left(g-P_{m-1}\right)_{t}(0)
$$

где $P_{m-1}-$ произвольный многочлен степени $m-1$.

Заметим, что

$$
\begin{aligned}
\frac{\partial}{\partial t} g_{t}(0)= & \int \sum_{j, k} a_{j}\left[c_{k}^{\prime}\left(r_{1}\right) r_{1}^{\prime} g\left(j r(t) v+\tau(t)\left(1+k r_{1}(t)\right)\right)\right. \\
& +c_{k}\left(r_{1}(t)\right) \sum_{i=1}^{n} D_{i} g\left(j r(t) v+\tau(t)\left(1+k r_{1}(t)\right)\right) \\
& \left.\times\left(j r^{\prime}(t) v_{i}+\tau_{i}^{\prime}(t)\left(1+k r_{1}(t)\right)+\tau_{i}(t) k r_{1}^{\prime}(t)\right)\right] \eta(v) d v
\end{aligned}
$$

Учитьвая, что

$$
D_{i} f(j r(t) v)=\frac{1}{j r(t)} \frac{\partial}{\partial v_{i}} f(j r(t) v),
$$

с помощью интегрирования по частям имеем

$$
\begin{aligned}
& \frac{\partial}{\partial t} g_{t}(0)=\int \sum_{j, k} a_{j} g\left(j r(t) v+\tau(t)\left(1+k r_{1}(t)\right)\right)\left\{c_{k}^{\prime}\left(r_{1}(t)\right) r_{1}^{\prime}(t) \eta(v)\right. \\
& \left.\quad-c_{k}\left(r_{1}(t)\right) \sum_{i=1}^{n}\left[\frac{r^{\prime}(t)}{r(t)} \eta(v)+\left(\frac{r^{\prime}(t)}{r(t)} v_{i}+\frac{\tau_{i}^{\prime}(t)\left(1+k r_{1}(t)\right)}{j r(t)}+\frac{\tau_{i}(t) k r_{1}^{\prime}(t)}{j r(t)}\right) D_{i} \eta(v)\right]\right\} d v .
\end{aligned}
$$

В силу (3.5) в правой части последнего равенства можно $g$ заменить на $g-P_{m-1}$, где $P_{m-1}(x)=\sum_{|\alpha| \leqslant m-1} c_{\alpha} x^{\alpha}-$ многочлен с произвольными коэффициентами.

Учитывая оценки $\left|r^{\prime}(t)\right| \leqslant c_{0},\left|\tau^{\prime}\right| \leqslant 1$ и оценки для $c_{k}(t), c_{k}^{\prime}(t)$, вытекающие из (3.4), получаем

$$
\begin{aligned}
\left|\frac{\partial}{\partial t} g_{t}(0)\right| & \leqslant C \int \sum_{j, k} r_{1}(t)^{-m+1} r(t)^{-1}\left|\left(g-P_{m-1}\right)\left(j r(t) v+\tau(t)\left(1+k r_{1}(t)\right)\right)\right| \chi(m v) d v \\
& \leqslant C_{1} \int \sum_{j, k} r_{1}(t)^{-m+1}\left|\left(g-P_{m-1}\right)(y)\right| r(t)^{-n-1} \chi\left(m \frac{y-\tau(t)-\tau(t) k r(t) / t}{j r(t)}\right) d y \\
& \leqslant C_{2} \int r_{1}(t)^{-m+1}\left|\left(g-P_{m-1}\right)(y)\right| r(t)^{-n-1} \chi\left(\frac{y-\tau(t)}{m r(t)}\right) d y \\
& =C_{2} \int t^{m-1}\left|\left(g-P_{m-1}\right)(y)\right| r(t)^{-m-n} \chi\left(\frac{y-\tau(t)}{m r(t)}\right) d y
\end{aligned}
$$

При этом коэффициенты многочлена $P_{m-1}$ могут зависеть от $t$. 
Заметим, что $g(t) \rightarrow g(0)$ при $t \rightarrow 0$, поскольку 0 - точка Лебега функции $g$. Это позволяет с помощью формулы Лейбница-Ньютона написать интегральное представление

$$
g(0)=-\int_{0}^{T} \frac{\partial}{\partial t} g_{t}(0) d t+g_{T}(0)
$$

с важной для дальнейшего оценкой (3.6) подьнтегрального выражения.

4. Класс рассматриваемых областей и поточечные оценки. Регулярной областью или областью с регулярной границей мы назьваем здесь область $G \subset \mathbb{R}^{n}$, гранища $\partial G$ которой локально представляет собой (после подходящего поворота декартовой системы координат) график функции, удовлетворяющей условию Липшица. Нашей целью является изучение пространств функций дробной гладкости, определенных на нерегулярной области.

Пусть $\rho(x)=\operatorname{dist}(x, \partial G), \rho_{1}=\min \{\rho(x), 1\}$, непрерывно дифференцируемая функция $r(x)$ эквивалентна $\rho_{1}(x)$ на $G, r(x) \leqslant \rho_{1}(x) / 2,|\operatorname{grad} r(x)| \leqslant 1$.

ОПРЕДЕЛЕНИЕ 4.1 [1], [2]. Пусть область $G \subset \mathbb{R}^{n}, \delta_{0} \in(0,1), R_{0}>0$ и каждой точке $x \in G$ поставлен в соответствие кусочно гладкий путь $\gamma=\gamma_{x}:\left[0, t_{x}\right] \rightarrow G$ со свойствами

$$
\gamma(0)=x, \quad \gamma \subset B\left(x, R_{0}\right), \quad \rho\left(\gamma\left(t_{x}\right)\right) \geqslant 2 \delta_{0}, \quad\left|\gamma^{\prime}\right| \leqslant 1 \quad \text { п.в., } \quad r\left(\gamma\left(t_{x}\right)\right) \geqslant \frac{1}{2} \delta_{0} .
$$

Тогда, положив

$$
H_{x}=\bigcup_{0 \leqslant t \leqslant t_{0}} B\left(\gamma_{x}(t), r\left(\gamma_{x}(t)\right)\right), \quad \mathscr{H}=\left\{H_{x}: x \in G\right\}
$$

будем писать $(G, \mathscr{H}) \in \mathscr{G}\left(\delta_{0}, R_{0}\right)$.

ЗАмЕЧАнИЕ 4.1 . Конструкции, подобные $\mathscr{G}\left(\delta_{0}, R_{0}\right)$, рассматривались в [15], а их обобщения - в [1], [2].

ОПРЕДЕЛЕНИЕ $4.2[16]$. Пусть область $G \subset \mathbb{R}^{n}, \delta_{0}>0, R_{0}>0,(G, \mathscr{H}) \in \mathscr{G}\left(\delta_{0}, R_{0}\right)$. Будем говорить, что $(G, \mathscr{H}) \in \mathscr{G}_{0}\left(\delta_{0}, R_{0}\right)$, если существует постоянная $C_{0}>0$ такая, что

$$
\int_{0}^{t_{x}} \frac{1}{r\left(\gamma_{x}(t)\right)} \chi\left(\frac{y-\gamma_{x}(t)}{r\left(\gamma_{x}(t)\right)}\right) d t \leqslant C_{0}
$$

для любых $y, x \in G$.

ЗАМЕчАниЕ 4.2 . В условиях определения 4.1 существует такое число $\kappa>0$, что

$$
\kappa r(x) \leqslant 3 r(y), \quad \text { если } x, y \in G, \quad B(x, r(x)) \cap B(y, r(y)) \neq \varnothing .
$$

В самом деле, достаточно учесть, как и в [15], что

$$
\rho_{1}(x) \leqslant \rho_{1}(y)+|x-y| \leqslant \rho_{1}(y)+r(x)+r(y) \leqslant \frac{3}{2} \rho_{1}(y)+\frac{1}{2} \rho_{1}(x),
$$

откуда $\rho_{1}(x) \leqslant 3 \rho_{1}(y)$. 
ОПРЕДЕЛЕНИЕ 4.3. При $\sigma \geqslant 1$ область $G \subset \mathbb{R}^{n}$ назовем областью $с$ условием гибкого $\sigma$-конуса, если при некоторых $t^{*}>0, \kappa>0$ для любого $x \in G$ существует кусочно гладкий путь $\gamma:\left[0, t^{*}\right] \rightarrow G, \gamma(0)=x,\left|\gamma^{\prime}\right| \leqslant 1$ п.в., такой, что $\rho(\gamma(t)) \geqslant \kappa t^{\sigma}$ при $0<t \leqslant t^{*}$.

ОПРЕДЕЛЕНИЕ 4.4 [15]. При $\sigma \geqslant 1$ область $G \subset \mathbb{R}^{n}$ называется областью с $\sigma$-условием Джсона ( $\sigma$-John domain), если существует точка $x^{0} \in G$ и постоянная $\kappa>0$ такие, что для любой точки $x \in G$ найдется спрямляемая кривая $\gamma:\left[0, l_{x}\right] \rightarrow G, \gamma(0)=x$, $\gamma\left(l_{x}\right)=x^{0}$, параметризованная с помощью длины дуги и такая, что

$$
\rho(\gamma(t)) \geqslant \kappa t^{\sigma} \quad \text { при } 0<t \leqslant l_{x} \text {. }
$$

Заметим, что для ограниченной области $G$ определения 4.3 и 4.4 эквивалентны.

Пусть теперь $(G, \mathscr{H}) \in \mathscr{G}\left(\delta_{0}, R_{0}\right), r_{\gamma}(t)=r(\gamma(t)), r(x) \leqslant \rho_{1}(x) / 2$. Зафиксируем $x \in G$, и пусть $\gamma=\gamma_{x}$ взято из определения 4.1. Построим исходя из $r(x)$ и $\gamma$ путь $\Gamma=\Gamma_{x}:\left[0, T_{x}\right] \rightarrow G$, задаваемые формулой

$$
\Gamma=\Gamma_{x}= \begin{cases}x & \text { при } 0 \leqslant t \leqslant r(x)=r_{\gamma}(0), \\ \gamma_{x}\left(t-r_{\gamma}(0)\right) & \text { при } r_{\gamma}(0) \leqslant t \leqslant t_{x}+r_{\gamma}(0)=T_{x},\end{cases}
$$

и непрерьвную, кусочно непрерьвно дифференцируемую функцию $r(t):\left(0, T_{x}\right] \rightarrow(0, \infty)$, задаваемую формулой

$$
r(t)=r_{\Gamma}(t)=r_{\Gamma_{x}}(t)= \begin{cases}t & \text { при } 0<t \leqslant r_{\gamma}(0), \\ r_{\gamma}\left(t-r_{\gamma}(0)\right) & \text { при } r_{\gamma}(0) \leqslant t \leqslant r_{\gamma}(0)+t_{x}=T_{x} .\end{cases}
$$

Пусть $f \in L(G, \mathrm{loc}), x \in G, 0<t \leqslant T_{x}$. Запишем усреднение (3.1) для функции $g(y)=f(x+y)$ при $\tau(t)=\Gamma(t)-x, r(t)$ из (4.4), (4.5) в виде

$$
f_{t}(x)=\int \Omega(y, \Gamma(t)-x, r(t)) f(x+y) d y .
$$

Тогда для почти каждого $x \in G$ по формуле Лейбница-Ньютона

$$
f(x)=-\int_{0}^{T_{x}} \frac{\partial}{\partial t} f_{t}(x) d t+f_{T_{x}}(x) .
$$

В силу (3.6)

$$
\begin{aligned}
|f(x)| \leqslant & C \int_{0}^{T_{x}} \int t^{m-1} r(t)^{-m-n} \chi\left(\frac{y+x-\Gamma_{x}(t)}{r(t)}\right)\left|f(x+y)-P_{m-1}(x, t ; y)\right| d y d t \\
& +\left|f_{T_{x}}(x)\right|
\end{aligned}
$$

где $P_{m-1}(x, t ; y)=\sum_{|\alpha| \leqslant m-1} c_{\alpha}(x, t) y^{\alpha}-$ многочлен с произвольными коэффициентами.

Без ограничения общности будем считать, что $\delta_{0}<1 / 2$. Тогда при $|z|<r(t)$

$$
B\left(\Gamma_{x}(t), \frac{r(t)}{2}\right) \subset \bar{B}\left(\Gamma_{x}(t)+z, r(t)\right) \subset G .
$$


Поэтому в $(4.7) \chi\left(2\left(y+x-\Gamma_{x}(t)\right) / r(t)\right)$ можно заменить на $\chi\left(\left(y+x-z-\Gamma_{x}(t)\right) / r(t)\right)$ и считать, что $P_{m-1}=P_{m-1}(x, t, z ; y)$ - многочлен по $y$ с коэффициентами, зависяшими от $x, t, z$. Выбрав его надлежашим образом, получим

$$
|f(x)| \leqslant C \int_{0}^{T_{x}} t^{m-1} r(t)^{-m} D_{m-1}(r(t), G) f\left(\Gamma_{x}(t)+z\right) d t+\left|f_{T_{x}}(x)\right|
$$

Усредняя эту оценку по $z \in B(0, r(t))$, получаем

$$
|f(x)| \leqslant C_{1} \int_{0}^{T_{x}} \int_{|z|<r(t)} t^{m-1} r(t)^{-m-n} D_{m-1}(r(t), G) f\left(\Gamma_{x}(t)+z\right) d z d t+\left|f_{T_{x}}(x)\right| .
$$

Введем при $s>0$ функцию

$$
\phi(x)=\sup _{0<t \leqslant \rho_{1}(x) / 2} t^{-s} D_{m-1}(t, G) f(x), \quad x \in G .
$$

Отметим сразу, что при любом $\theta \in[1, \infty]$ вьполняется оценка

$$
\phi(x) \leqslant C \phi_{\theta}(x)=C\left(\int_{0}^{\rho_{1}(x)}\left[\frac{D_{m-1}(t, G) f(x)}{t^{s}}\right]^{\theta} \frac{d t}{t}\right)^{1 / \theta}
$$

В самом деле, при $\theta=\infty$ оценка (4.9) очевидна. Если же $1 \leqslant \theta<\infty$ и

$$
t_{0}^{-s} D_{m-1}\left(t_{0}, G\right) f(x) \geqslant \frac{\phi(x)}{2}
$$

то оценка (4.9) следует из оценки

$$
\phi(x) \leqslant C\left(\int_{t_{0}}^{2 t_{0}}\left[\frac{D_{m-1}(t, G) f(x)}{t^{s}}\right]^{\theta} \frac{d t}{t}\right)^{1 / \theta} .
$$

Последняя оценка очевидна при замене в определении $D_{m-1}(t, G) f$ приближения с помощью проекционного оператора на наилучшее приближение, а значит, и в приведенном виде в силу замечания 1.1.

Из (4.8) имеем

$$
|f(x)| \leqslant C_{1} \int_{0}^{T_{x}} \int_{\left|y-\Gamma_{x}(t)\right|<r(t)} t^{m-1} r(t)^{s-m-n} \phi(y) d y d t+\left|f_{T_{x}}(x)\right| .
$$

ЗАМЕчАниЕ 4.3. Можно ожидать, что при $1 \leqslant \theta<\tau \leqslant \infty$

$$
\phi_{\tau}(x) \leqslant C(\tau, \theta) \phi_{\theta}(x)
$$


Лемма 4.1. Пусть $s>0,(G, \mathscr{H}) \in \mathscr{G}\left(\delta_{0}, R_{0}\right), x \in G$, nymb $\gamma=\gamma_{x}:\left[0, t_{x}\right] \rightarrow G u$ функиия $r(x)$ те же, что в определении 4.1. Тогда если $f \in L(G, \operatorname{loc})$ и $x$ - точка Лебега функиии $f$, то

$$
|f(x)| \leqslant C A_{1} \phi(x)+C A_{2} \phi(x)+C A_{3} f(x),
$$

$2 \partial e$

$$
\begin{aligned}
& A_{1} \phi(x)=\int_{|y-x|<r(x)} \frac{\phi(y) d y}{|y-x|^{n-s}} \leqslant \int_{G \cap B(x, 1)} \frac{\phi(y) d y}{|y-x|^{n-s}} \\
& A_{2} \phi(x)=\int_{0}^{t_{x}}(t+r(x))^{m-1} r(\gamma(t))^{s-m-n} \int_{|y-\gamma(t)|<r(\gamma(t))} \phi(y) d y d t \\
& A_{3} f(x)=\int_{\left|y-\gamma\left(t_{x}\right)\right|<r\left(\gamma\left(t_{x}\right)\right)}|f(y)| d y \leqslant \int_{G_{\delta_{0}} \cap B\left(x, R_{0}+1\right)}|f(y)| d y .
\end{aligned}
$$

При этом постоянная $C$ не зависит от $f$ u $x$.

ДокАЗАТЕЛЬСТво следует непосредственно из (4.8), если интеграл по $\left[0, T_{x}\right]$ в $(4.8)$ разбить на два интеграла (по $[0, r(x)]$ и $\left.\left[r(x), T_{x}\right]\right)$, учесть $(4.4),(4.5)$ и заменить во втором из интегралов переменную $t$ на $t+r(x)$. Лемма доказана.

5. Оценки слабого типа. Пусть $1 \leqslant p<q<\infty$. Говорят, что оператор $A$ имеет сильный тип $(p, q)$, если

$$
\left\|A f\left|L_{q}(G)\|\leqslant C\| f\right| L_{p}(G)\right\| \quad \forall f \in L_{p}(G)
$$

и что $A$ имеет слабый тuп $(p, q)$, если

$$
\sup _{\lambda>0} \lambda|\{x \in G:|A f(x)|>\lambda\}|^{1 / q} \leqslant C\left\|f \mid L_{p}(G)\right\| \quad \forall f \in L_{p}(G) .
$$

Эти две оценки называются соответственно оценками сильного и слабого типа. Оценка сильного типа влечет оценку слабого типа, но не наоборот. Однако в некоторых случаях с помощью оценок слабого типа можно получить оценку сильного типа, используя, например, интерполяционную теорему Марцинкевича или другие соображения.

Лемма 5.1. Пусть $1 \leqslant p<q<\infty, s-n / p+n / q \geqslant 0$. Тогда оператор $A_{1}$ имеет слабый тип $(p, q)$. Если же при әтом $p>1$ или $s-n / p+n / q>0$, то $A_{1}$ имеет сильный muп $(p, q)$.

ДокАЗАТЕЛЬСТВО при $s-n / p+n / q=0$ является следствием классических оценок для потенциала Рисса (см., например, [17, гл. 5]). При $s-n / p+n / q>0$ достаточно воспользоваться неравенством Юнга для сверток.

Лемма 5.2. При $1 \leqslant r \leqslant q<\infty$ оператор $A_{3}$ имеет сильный тип $(r, q)$. 
ДокАЗАТЕЛЬСтво следует из неравенства Юнга.

Обозначим через $M \phi$ максимальную функцию для функции $\phi$. Введем для $x \in G$

$$
\begin{aligned}
N(p, q, x)= & \sup _{R>0}\left[\int_{0}^{t_{x}} \chi\left(\frac{R}{t+r(\gamma(t))}\right)(t+r(x))^{(m-1) p^{\prime}}\right. \\
& \left.\times r(\gamma(t))^{(s-m+(1-n) / p) p^{\prime}} d t\right]^{1 / p^{\prime}}|B(x, R)|^{1 / q}, \\
N(p, q, G)= & \sup _{x \in G} N(p, q, x) .
\end{aligned}
$$

ЛЕмма 5.3 (основная). Пусть

$$
\begin{array}{cl}
(G, \mathscr{H}) \in \mathscr{G}_{0}\left(\delta_{0}, R_{0}\right), & s>0, \quad 1 \leqslant p_{0} \leqslant p<q<\infty, \quad \frac{1}{p_{0}}-\frac{1}{q_{0}}=\frac{1}{p}-\frac{1}{q}, \\
& N(p, q, G)+N\left(p_{0}, q_{0}, G\right)<\infty .
\end{array}
$$

Тогда существует постоянная $C>0$ такая, что для всех $x \in G$

$$
A_{2} \phi(x) \leqslant C N(p, q, x)\left\|\phi \mid L_{p}(G)\right\|^{1-p / q}\left(M\left(|\phi|^{p_{0}}\right)(x)\right)^{p /\left(p_{0} q\right)}
$$

для любой функиии ф с конечной правой частью.

ДоКАЗАТЕЛЬСтво. Достаточно воспользоваться леммой 1 из [18].

СледСтвиЕ 5.1. В условиях леммы 5.3 при $1 \leqslant p_{0}<p$ оператор $A_{2}$ имеет сильный тип $(p, q)$, а при $1 \leqslant p_{0}=p$ слабый тип $(p, q)$.

6. Теоремы вложения. Здесь будут приведены некоторые теоремы вложения пространств функций положительной гладкости, определенных на области $G \subset \mathbb{R}^{n}$ с нерегулярной границей, в пространство Лебега $L_{q}(G)$. Но начнем мы с леммы о вложении в пространство Марцинкевича, содержащее $L_{q}(G)$, которая является основой для всех последующих вложений.

Везде в дальнейшем будем считать, что

$$
m \in \mathbb{N}, \quad 0<s<m, \quad 1 \leqslant p<q<\infty, \quad 1 \leqslant r \leqslant q, \quad s-\frac{n}{p}+\frac{n}{q} \geqslant 0, \quad 1 \leqslant \theta \leqslant \infty .
$$

ЛЕмма 6.1. Пусть выполнены условия (6.1), область $G \subset \mathbb{R}^{n},(G, \mathscr{H}) \in$ $\mathscr{G}\left(\delta_{0}, R_{0}\right)$

$$
N(p, q, G)<\infty
$$

Тогда

$$
\sup _{\lambda>0} \lambda|\{x \in G:|f(x)|>\lambda\}|^{1 / q} \leqslant C\left\|f \mid L_{p \theta, r}^{s(m)}(G)\right\|,
$$

где C не зависит от функиии $f$ с конечной правой частью (6.3). 
ДоКАЗАТЕЛЬСТвО. Каждый из усеченных гибких конусов $H_{x}$ из условия леммы можно заменить “правильньм" усеченньм гибким конусом $\widehat{H}_{x} \subset H_{x}$ таким, что для построенной по $\widehat{H}_{x}$ левой части (5.1) (обозначим ее через $\widehat{N}(p, q, x)$ ) окажется верной оценка

$$
\widehat{N}(p, q, x) \leqslant C N(p, q, x),
$$

где $C$ не зависит от $x \in G$. Способ такого “исправления" $H_{x}$ предложен в [15]. В [2], [16] он применен к случаю, который отличается от нашего формальной заменой в $N(p, q, x)$ параметра $s$ на $m$. В нашем случае оно проводится так же, как в [2]. Тем самьм, без ограничения общности будем считать, что в условиях леммы $\mathscr{G}\left(\delta_{0}, R_{0}\right)$ заменено на $\mathscr{G}_{0}\left(\delta_{0}, R_{0}\right)$.

Используя оценку (4.11), леммы 5.1, 5.2 и следствие 5.1, приходим к оценке

$$
\sup _{\lambda>0} \lambda|\{x \in G:|f(x)|>\lambda\}|^{1 / q} \leqslant C\left[\left\|\phi\left|L_{p}(G)\|+\| f\right| L_{r}\left(G_{\delta}\right)\right\|\right]
$$

где функция $\phi$ та же, что в (4.9), а постоянная $C$ не зависит от $f$.

В последней оценке можно в силу (4.9) заменить $\phi$ на $C_{1} \phi_{0}$, что и приводит к $(6.3)$.

СлЕДСТВИЕ 6.1. В условиях леммы 6.1 справедлива оценка

$$
\sup _{\lambda>0} \lambda|\{x \in G:|f(x)|>\lambda\}|^{1 / q} \leqslant C\left\|f \mid B_{p p, r}^{s(m)}(G)\right\|
$$

поскольку

$$
\left\|f\left|B_{p p, r}^{s(m)}(G)\|=\| f\right| L_{p p, r}^{s(m)}(G)\right\|
$$

ТЕОрема 6.1. Пусть в условия леммы 6.1 имеет место условие $0<s<m=1$. Тогда при $1 \leqslant \theta \leqslant p$ выполнены оченки

$$
\begin{aligned}
& \left\|f\left|L_{q}(G)\|\leqslant C\| f\right| L_{p \theta, r}^{s}(G)\right\|, \\
& \left\|f\left|L_{q}(G)\|\leqslant C\| f\right| B_{p p, r}^{s}(G)\right\|,
\end{aligned}
$$

где $C$ не зависит от функиии $f$ с конечной правой частью (6.5) или (6.6) соответственно.

ДокАЗАтЕльСтво. Рассмотрим функцию $\phi \in C_{0}^{\infty}\left(\mathbb{R}^{1}\right)$, удовлетворяющую условиЯM

$$
0 \leqslant \phi \leqslant 1, \quad \operatorname{supp} \phi \subset\left(\frac{1}{2}, 2\right), \quad \sum_{-\infty}^{\infty} \phi\left(2^{-k} t\right)=1,
$$

$f_{k}(x)=\phi\left(2^{-k}|f(x)|\right) f(x)$, так что

$$
f(x)=\sum_{-\infty}^{\infty} f_{k}(x)
$$

Введем еще

$$
E_{k}=\left\{x \in G:|f(x)|>2^{k}\right\}, \quad E_{k}^{*}=\left\{x \in G:\left|f_{k}(x)\right|>2^{-k-2}\right\} .
$$


Тогда так же, как в [2], имеем

$$
\begin{aligned}
I^{q} & =\int_{G}|f(x)|^{q} d x+\sum_{k \in \mathbb{Z}} \int_{E_{k} \backslash E_{k+1}}|f(x)|^{q} d x \\
& \leqslant \sum_{k} \int_{E_{k} \backslash E_{k-1}}\left|f_{k}(x)+f_{k+1}(x)\right|^{q} d x \\
& \leqslant \sum_{k} 2^{(k+1) q}\left|\left\{x \in G:\left|f_{k}(x)+f_{k+1}(x)\right|>2^{k}\right\}\right| \\
& \leqslant \sum_{k} 2^{(k+1) q}\left(\left|E_{k}^{*}\right|+\left|E_{k+1}^{*}\right|\right)=2^{2 q}\left(2^{q}+1\right) \sum_{k} 2^{(k-2) q}\left|E_{k}^{*}\right| .
\end{aligned}
$$

Используя теорему 6.1 , получаем отсюда, что

$$
\begin{aligned}
I^{q} & \leqslant C \sum_{k}\left\|f_{k} \mid L_{p \theta, r}^{s}(G)\right\|^{q} \\
& \leqslant C_{1}\left(\sum_{k}\left\|f_{k} \mid l_{p \theta}^{s}(G)\right\|^{p}\right)^{q / p}+C_{1}\left(\sum_{k}\left\|f_{k} \mid L_{r}\left(G_{\delta}\right)\right\|^{r}\right)^{q / r} \\
& \leqslant C_{1}\left(\sum_{k}\left\|f_{k} \mid l_{p \theta}^{s}(G)\right\|^{p}\right)^{q / p}+C_{2}\left\|f \mid L_{r}\left(G_{\delta}\right)\right\|^{q} .
\end{aligned}
$$

Покажем, что

$$
\sum_{k}\left\|f_{k}\left|l_{p \theta}^{s}(G)\left\|^{p} \leqslant C\right\| f\right| l_{p \theta}^{s}(G)\right\|^{p} .
$$

Зафиксируем $x, h$. Предполагая, что $|f(x+h)| \geqslant|f(x)|$, имеем

$$
\begin{aligned}
& \left|f_{k}(x+h)-f_{k}(x)\right|=\left|\phi\left(2^{-k}|f(x+h)|\right) f(x+h)-\phi\left(2^{-k}|f(x)|\right) f(x)\right| \\
& \quad \leqslant \phi\left(2^{-k}|f(x+h)|\right)|f(x+h)-f(x)|+|f(x)| \cdot\left|\phi\left(2^{-k}|f(x+h)|\right)-\phi\left(2^{-k}|f(x)|\right)\right| \\
& \quad \leqslant \phi\left(2^{-k}|f(x+h)|\right)|f(x+h)-f(x)|+|f(x)| 2^{-k}\left|\int_{|f(x)|}^{|f(x+h)|} \phi^{\prime}\left(2^{-k} t\right) d t\right| \\
& \quad \leqslant \phi\left(2^{-k}|f(x+h)|\right)|f(x+h)-f(x)|+\int_{|f(x)|}^{|f(x+h)|} 2^{-k} t\left|\phi^{\prime}\left(2^{-k} t\right)\right| d t .
\end{aligned}
$$

Аналогичная оценка получается и при $|f(x+h)|<|f(x)|$.

Отсюда

$$
\begin{aligned}
\int_{0}^{1}\left|f_{k}(x+h)-f_{k}(x)\right|^{\theta} \frac{d h}{h^{s \theta+1}} & \\
\leqslant & 2^{\theta} \int_{0}^{1}\left[\phi\left(2^{-k}|f(x+h)|\right)+\phi\left(2^{-k}|f(x)|\right)\right]^{\theta}|f(x+h)-f(x)|^{\theta} \frac{d h}{h^{s \theta+1}} \\
& +C \int_{0}^{1}\left|\int_{|f(x)|}^{|f(x+h)|} d t\right|^{\theta / \theta^{\prime}} \int_{|f(x)|}^{|f(x+h)|}\left|\phi^{\prime}\left(2^{-k} t\right)\right|^{\theta} d t \frac{d h}{h^{s \theta+1}} .
\end{aligned}
$$


При $\theta \leqslant p$ имеем

$$
\begin{aligned}
& \sum_{k}\left\|f_{k} \mid l_{p \theta}^{s}(G)\right\|^{p} \leqslant C \int_{G}\left(\int_{0}^{1} \sum_{k}\left|f_{k}(x+h)-f_{k}(x)\right|^{\theta} \frac{d h}{h^{s \theta+1}}\right)^{p / q} d x \\
& \leqslant C_{1} \int_{G}\left(\int_{0}^{1}|f(x+h)-f(x)|^{\theta} \frac{d h}{h^{s \theta+1}}\right)^{p / \theta} d x \\
&+C_{1} \int_{G}\left(\int_{0}^{1}|f(x+h)-f(x)|^{\theta / \theta^{\prime}}|f(x+h)-f(x)| \frac{d h}{h^{s \theta+1}}\right)^{p / \theta} d x \\
& \leqslant C_{2} \int_{G}\left(\int_{0}^{1}|f(x+h)-f(x)|^{\theta} \frac{d h}{h^{s \theta+1}}\right)^{p / \theta} d x=C_{2}\left\|f \mid l_{p \theta}^{s}(G)\right\|^{p}
\end{aligned}
$$

и оценка (6.8) установлена.

Из (6.8) и (6.7) следует (6.5). Оценка же (6.6) совпадает со случаем $\theta=p$ оценки (6.5). Теорема доказана.

ТЕОРема 6.2. В условиях леммы 6.1 при $1 \leqslant \theta \leqslant p<\widetilde{q}<q$ существует постоянная $C>0$ такая, что имеют место неравенства

$$
\begin{aligned}
& \left\|f\left|L_{\widetilde{q}}(G)\|\leqslant C\| f\right| L_{p \theta, r}^{s(m)}(G)\right\| \\
& \left\|f\left|L_{\widetilde{q}}(G)\|\leqslant C\| f\right| B_{p p, r}^{s(m)}(G)\right\|
\end{aligned}
$$

для всех функиий $f$ с конечной правой частью (6.9) или (6.10) соответственно.

ДокАЗАТЕЛЬСтво. Оценка (6.10) совпадает с (6.9) при $\theta=p$ в силу (6.4). Установим (6.9). С помощью лемм 4.1, 5.1-5.3 доказательство (6.9) сводится к оценке нормы оператора

$$
A_{2}: L_{p}(G) \rightarrow L_{\widetilde{q}}(G)
$$

с использованием (5.3). Эта оценка устанавливается так же, как утверждение іiі) леммы 4.2 из [2].

ТЕОРема 6.3. Пусть $1<p<q<\infty$ и выполнены условия леммы 6.1 с заменой (6.2) на

$$
N\left(p_{0}, q_{0}, G\right)+N\left(p_{1}, q_{1}, G\right)<\infty,
$$

əде $1 \leqslant p_{j}<q_{j}<\infty, j=0,1, q_{0} \neq q_{1}$,

$$
\frac{1}{p}=\frac{1-\mu}{p_{0}}+\frac{\mu}{p_{1}}, \quad \frac{1}{q}=\frac{1-\mu}{q_{0}}+\frac{\mu}{q_{1}}, \quad 0<\mu<1 .
$$

Тогда при $1 \leqslant \theta \leqslant p$ выполнень оценки

$$
\begin{aligned}
& \left\|f\left|L_{q}(G)\|\leqslant C\| f\right| L_{p \theta, r}^{s(m)}(G)\right\|, \\
& \left\|f\left|L_{q}(G)\|\leqslant C\| f\right| B_{p p, r}^{s(m)}(G)\right\|,
\end{aligned}
$$

где $C$ не зависит от функиии $f$. 
ДокАЗАТЕльство. Оценка (6.12) в силу (6.4) совпадает с (6.11) при $\theta=p$. Для доказательства (6.11) в силу (4.11) и (4.9) достаточно установить ограниченность операторов

$$
A_{1}: L_{p}(G) \rightarrow L_{q}(G), \quad A_{2}: L_{p}(G) \rightarrow L_{q}(G), \quad A_{3}: L_{r}(G) \rightarrow L_{q}\left(G_{\delta}\right),
$$

считая при этом, что $(G, \mathscr{H}) \in \mathscr{G}_{0}\left(\delta_{0}, R_{0}\right)$ (обоснование последнего см. в начале доказательства леммы 6.1).

Ограниченность операторов $A_{1}$ и $A_{3}$ следует соответственно из лемм 5.1 и 5.2.

Оператор $A_{2}$ по следствию 5.1 имеет слабый тип $\left(p_{0}, q_{0}\right)$ и слабый тип $\left(p_{1}, q_{1}\right)$. Поэтому в силу интерполяционной теоремы Марцинкевича он имеет сильньй тип $(p, q)$. Теорема доказана.

ТЕОРемА 6.4. Пусть в условиях леммы 6.1 выполнены следующие соотношения параметров:

$$
\begin{gathered}
1 \leqslant p_{0}<p<q<\infty, \quad \frac{1}{p_{0}}-\frac{1}{q_{0}}=\frac{1}{p}-\frac{1}{q}, \quad 1 \leqslant r \leqslant q, \quad s-\frac{n}{p}+\frac{n}{q} \geqslant 0, \\
N(p, q, G)+N\left(p_{0}, q_{0}, G\right)<\infty .
\end{gathered}
$$

Тогда при $1 \leqslant \theta \leqslant$ справедливы оценки (6.11), (6.12).

ДокАЗАТЕЛЬСтво. то же, что и в теореме 6.3 , с тем лишш отличием, что ограниченность оператора $A_{2}$ устанавливается с помощью следствия 5.1 без использования интерполяционной теоремы Марцинкевича.

ТЕОремА 6.5. Пусть для области $G$ с условием гибкого б-конуса, $\sigma \geqslant 1$ (см. определение 4.3), выполнены условия (6.1),

$$
\begin{gathered}
1<p<q<\infty \quad(1 \leqslant p<q<\infty \quad \text { npu } \quad 0<s<m=1), \quad 1 \leqslant r \leqslant q \\
s-(m-s)(\sigma-1)-\frac{\sigma(n-1)+1}{p}+\frac{n}{q} \geqslant 0 .
\end{gathered}
$$

Тогда при $1 \leqslant \theta \leqslant p$ справедливы оценки (6.11), (6.12). ${ }^{1}$

ДокАЗАтЕЛЬСтво. Проверим выполнение условия (6.2) при $0<t<t_{x}=t^{*}$, $2 r(\gamma(t)) \geqslant \kappa t^{\sigma}$. Для этого достаточно показать, что

$$
\sup _{x \in G} \sup _{R>0}\left[I_{1}(x, R)+I_{2}(x, R)\right]<\infty
$$

где

$$
\begin{gathered}
I_{1}(x, R)=\left(\int_{0}^{\tau_{x}} \chi\left(\frac{R}{3 \rho_{1}(x)}\right) \rho_{1}(x)^{(s-1+(1-n) / p) p^{\prime}} d t\right)^{1 / p^{\prime}} R^{n / q} \\
I_{2}(x, R)=\left(\int_{\tau_{x}}^{t^{*}} \chi\left(\frac{R}{3 t}\right) t^{(m-1) p^{\prime}} t^{\sigma(s-m+(1-n) / p) p^{\prime}} d t\right)^{1 / p^{\prime}} R^{n / q} \\
\tau_{x}=\min \left\{t^{*}, \rho_{1}(x)\right\}
\end{gathered}
$$

\footnotetext{
1Эта теорема приведена в [19] с опечаткой в формуле (12). Там следует заменить $s$ на $\sigma s$, после чего она совпадет с (6.13).
} 
Ho

$$
\begin{aligned}
I_{1}(x, R) \leqslant & C_{1} \chi\left(\frac{R}{3 \rho_{1}(x)}\right) \rho_{1}(x)^{s-n / p+n / q} \leqslant C_{1}<\infty \\
& I_{2}(x, R)=0 \quad \text { при } \rho_{1}(x) \geqslant t^{*}
\end{aligned}
$$

а при $\tau_{x}=\rho_{1}(x) / C_{0}<t^{*}$ имеем следующее.

В случае, когда $m-\sigma(m-s+(n-1) / p)-1 / p<0$, имеем

$$
I_{2}(x, R) \leqslant C \chi\left(\frac{R}{3 t^{*}}\right)\left(\rho_{1}(x)+R\right)^{m-\sigma(m-s+(n-1) / p)-1 / p} R^{n / q} \leqslant C_{1} .
$$

В случае, когда $m-\sigma(m-s+(n-1) / p)-1 / p>0$, имеем

$$
I_{2}(x, R) \leqslant C \chi\left(\frac{R}{3 t^{*}}\right)\left(t^{*}\right)^{m-\sigma(m-s+(n-1) / p)-1 / p} \leqslant C_{2} .
$$

В случае, когда $m-\sigma(m-s+(n-1) / p)-1 / p=0$, имеем

$$
I_{2}(x, R) \leqslant C \chi\left(\frac{R}{3 t^{*}}\right)\left(\ln \frac{3 t^{*}}{R}\right) R^{n / q} \leqslant C_{3} .
$$

Из оценок, полученных для $I_{1}(x, R), I_{2}(x, R)$, вытекает, что условие (6.1) вьполнено.

Пусть сначала $0<s<m=1$. Тогда утверждение теоремы вьполняется в силу теоремы 6.1.

Пусть теперь $p>1$. Выберем $p_{0}, p_{1}, q_{0}, q_{1}$ таким образом, чтобы $1 \leqslant p_{j}<q_{j}<\infty$, $j=0,1, p_{0}<p_{1}, q_{0}<q_{1}$,

$$
\frac{1}{2 p_{0}}+\frac{1}{2 p_{1}}=\frac{1}{p}, \quad \frac{1}{2 q_{0}}+\frac{1}{2 q_{1}}=\frac{1}{q} .
$$

Тогда левая часть (6.13) сохраняется при замене $(p, q)$ на $\left(p_{0}, q_{0}\right)$ и на $\left(p_{1}, q_{1}\right)$. В силу теоремы 6.3 получаем оценки (6.11), (6.12).

В качестве примера применения доказанных теорем рассмотрим область, имеющую вид пика $\left(x^{\prime}=\left(x_{1}, \ldots, x_{n-1}\right)\right)$ :

$$
\begin{gathered}
G=\left\{x=\left(x^{\prime}, x_{n}\right): 0<x_{n}<l+\psi(l) ;\left|x^{\prime}\right|<\psi\left(x_{n}\right) \text { при } 0<x_{n}<l\right. \\
\left.\left|x^{\prime}\right|^{2}+\left|x_{n}-l\right|^{2}<\psi(l)^{2} \text { при } l \leqslant x_{n}<l+\psi(l)\right\}
\end{gathered}
$$

где функция $\psi$ на $[0, l]$ не убьвает и удовлетворяет условию Липшища, $\psi(0)=0, \psi>0$ на $(0, l]$. Будем считать, что при некотором $c>0$

$$
\psi(t) \leqslant c \psi\left(\frac{t}{2}\right) \quad \text { при } 0<t \leqslant l .
$$

Примем также чисто техническое ограничение

$$
\psi(t) \leqslant \frac{1}{2} t \quad \text { при } \quad 0<t \leqslant l .
$$


Пусть $\delta_{0}$ достаточно мало. При $x \in G, x_{n}<l$ положим

$$
\begin{array}{ll}
\gamma(t)=\gamma_{x}(t)=\left(x^{\prime}\left(1-\frac{t}{\left|x^{\prime}\right|}\right), x_{n}\right) & \text { при } 0<t \leqslant\left|x^{\prime}\right|, \\
\gamma(t)=\left(0^{\prime}, x_{n}+t-\left|x^{\prime}\right|\right) & \text { при }\left|x^{\prime}\right| \leqslant t \leqslant l-x_{n}+\left|x^{\prime}\right|=t_{x} .
\end{array}
$$

При $x \in G, x_{n} \geqslant l$ положим

$$
\begin{aligned}
\gamma(t) & =\gamma_{x}(t) \\
& =x+\frac{\left(0^{\prime}, l\right)-x}{\left(\left|x^{\prime}\right|^{2}+\left(x_{n}-l\right)^{2}\right)^{1 / 2}} t \quad \text { при } 0<t<\left(\left|x^{\prime}\right|^{2}+\left(x_{n}-l\right)^{2}\right)^{1 / 2}=t_{x}-\delta_{0}, \\
\gamma(t) & =\gamma_{x}(t)=\left(0^{\prime}, l\right)
\end{aligned}
$$

Введем

$$
\begin{gathered}
N^{*}(p, q, G)=\sup _{0<h \leqslant l} \sup _{0<d \leqslant \xi(h)}\left(\int_{d+h}^{1} t^{(m-1) p^{\prime}} \psi(t)^{(s-m(1-n) / p) p^{\prime}} d t\right)^{1 / p^{\prime}} \\
\times\left(\int_{d}^{d+h} \psi(t)^{n-1} d t\right)^{1 / q}
\end{gathered}
$$

где

$$
\xi(h)=\sup \left\{u: 0<u \leqslant l, \int_{u}^{u+h} \psi(t)^{n-1} d t \leqslant h^{n}\right\} .
$$

ТЕОРема 6.6. Пусть область $G \subset \mathbb{R}^{n}$ имеет вид пика (6.14), выполнены условия (6.1), $0<s<m=1 u$

$$
N^{*}(p, q, G)<\infty
$$

Тогда справедливы оченки (6.11), (6.12).

ТЕОРемА 6.7. Пусть область $G \subset \mathbb{R}^{n}$ имеет вид пика (6.14), выполнены условия $(6.1) c\left(p_{j}, q_{j}\right)$ вместо $(p, q)$ и условия

$$
N^{*}\left(p_{j}, q_{j}, G\right)<\infty, \quad j=0,1
$$

2วe

$$
\frac{1}{p}=\frac{1-\theta}{p_{0}}+\frac{\theta}{p_{1}}, \quad \frac{1}{q}=\frac{1-\theta}{q_{0}}+\frac{\theta}{q_{1}}, \quad 0<\theta<1, \quad q_{0} \neq q_{1} .
$$

Тогда справедливы оценки (6.11), (6.12). 
ДокАЗАТЕЛЬСТвО ТЕОРем $6.6,6.7$. Возьмем $\delta \in(0,1 / 2], r(x)=\delta \rho(x)$. Пусть сначала $x \in G, x_{n}<l$. Полагая $\rho=\rho(x)$, имеем (см. (5.1))

$$
\begin{aligned}
N(p, q, x) \leqslant & C_{1} \sup _{0<h \leqslant l}\left(\int_{0}^{t_{x}} \chi\left(\frac{h}{t+\delta_{0} \rho(\gamma(t))}\right)(t+\rho)^{(m-1) p^{\prime}}\right. \\
& \left.\times \rho(\gamma(t))^{(s-m(1-n) / p) p^{\prime}} d t\right)^{1 / p^{\prime}}|G \cap B(x, h)|^{1 / q} \\
= & C_{1} N_{0}(p, q, x) \leqslant C_{1} \sum_{j=1}^{3} N_{j}(p, q, x),
\end{aligned}
$$

где $N_{j}, j=1,2,3$, отличаются от $N_{0}$ лишь заменой отрезка интегрирования $\left[0, t_{x}\right]$ на его части соответственно $\left[0, \tau_{x}\right],\left[\tau_{x},\left|x^{\prime}\right|\right]$, $\left[\left|x^{\prime}\right|, t_{x}\right]$ при $\tau_{x}=\min \left\{\rho,\left|x^{\prime}\right|\right\}$ (если $\rho \geqslant\left|x^{\prime}\right|$, то второй отрезок вырождается в точку).

Заметим, что $\rho(\gamma(t))$ не убывает и что

$$
\rho \leqslant \rho(\gamma(t)) \leqslant \rho+|\gamma(t)-\gamma(0)| \leqslant \rho+t
$$

Имеем

$$
\begin{aligned}
N_{1} & \leqslant C_{2} \sup _{0<h \leqslant l} \chi\left(\frac{h}{\left(1+2 \delta_{0}\right) \rho}\right)\left(\int_{0}^{\tau_{x}} \rho^{(m-1) p^{\prime}} \rho^{(s-m+(1-n) / p) p^{\prime}} d t\right)^{1 / p^{\prime}} h^{n / q} \\
& \leqslant C_{3} \sup _{0<h \leqslant l} \chi\left(\frac{h}{\left(1+2 \delta_{0}\right) \rho}\right) \rho^{s-n / p} h^{n / q} \leqslant C_{4} \rho^{s-n / p+n / q} \leqslant C
\end{aligned}
$$

Если отрезок $\left[\tau_{x},\left|x^{\prime}\right|\right]$ не вырождается в точку, то, учитьвая, что $\rho(\gamma(t))$ растет на нем не медленнее некоторой линейной функции с положительной производной, имеем

$$
\begin{aligned}
N_{2} & \leqslant C_{5} \sup _{0<h \leqslant l}\left(\int_{\rho}^{\left|x^{\prime}\right|} \chi\left(\frac{h}{\left(1+2 \delta_{0}\right) t}\right) t^{(s-n / p) p^{\prime}-1} d t\right)^{1 / p^{\prime}} h^{n / q} \\
& \leqslant C_{6} \sup _{0<h \leqslant l} \chi\left(\frac{h}{\left(1+2 \delta_{0}\right)\left|x^{\prime}\right|}\right) h^{n / q}\left\{\begin{array}{ll}
h^{s-n / p} & \text { при } s-\frac{n}{p}<0 \\
\ln \frac{\left|x^{\prime}\right|}{h} & \text { при } s-\frac{n}{p}=0 \\
\left|x^{\prime}\right|^{s-n / p} & \text { при } s-\frac{n}{p}>0
\end{array}\right\} \leqslant C .
\end{aligned}
$$

Имеем далее

$$
\begin{aligned}
N_{3} \leqslant & C_{7} \sup _{0<h \leqslant l}\left(\int_{\left|x^{\prime}\right|}^{l-x_{n}+\left|x^{\prime}\right|} \chi\left(\frac{h}{\left(1+2 \delta_{0}\right) t}\right) t^{(m-1) p^{\prime}}\right. \\
& \left.\times \psi\left(x_{n}+t-\left|x^{\prime}\right|\right)^{(s-m+(1-n) / p) p^{\prime}} d t\right)^{1 / p^{\prime}}|G \cap B(x, h)|^{1 / q} \\
\leqslant & C_{8} \sup _{0<h \leqslant l}\left(\int_{x_{n}}^{l} \chi\left(\frac{h}{\left(1+2 \delta_{0}\right)\left(u+\left|x^{\prime}\right|-x_{n}\right)}\right)\left(u+\left|x^{\prime}\right|-x_{n}\right)^{(m-1) p^{\prime}}\right. \\
& \left.\times \psi(u)^{(s-m+(1-n) / p) p^{\prime}} d u\right)^{1 / p^{\prime}}\left(\min \left\{h^{n}, \int_{x_{n}}^{x_{n}+h} \psi(t)^{n-1} d t\right\}\right)^{1 / q} .
\end{aligned}
$$


Учитьвая (6.16), заменим под знаком интеграла $\left|x^{\prime}\right|$ на $x_{n}$. Тогда

$$
\begin{aligned}
N_{3} \leqslant & C_{9} \sup _{0<h \leqslant l}\left(\int_{\max \left\{x_{n}, h / 2\right\}}^{l} t^{(m-1) p^{\prime}} \psi(t)^{(s-m+(1-n) / p) p^{\prime}} d t\right)^{1 / p^{\prime}} \\
& \times\left(\min \left\{h^{n}, \int_{x_{n}}^{x_{n}+h} \psi(t)^{n-1} d t\right\}\right)^{1 / q} \cdot
\end{aligned}
$$

Поскольку $\max \left\{x_{n}, h / 2\right\} \geqslant\left(x_{n}+h\right) / 3$, используя $(6.15)$, имеем

$$
\begin{aligned}
N_{3} \leqslant & C_{10} \sup _{0<h \leqslant l}\left(\int_{\left(x_{n}+h\right) / 3}^{l} t^{(m-1) p^{\prime}} \psi(t)^{(s-m+(1-n) / p) p^{\prime}} d t\right)^{1 / p^{\prime}} \\
& \times\left(\min \left\{\left(\frac{h}{3}\right)^{n}, \int_{x_{n} / 3}^{\left(x_{n}+h\right) / 3} \psi(t)^{n-1} d t\right\}\right)^{1 / q} \\
\leqslant & C_{11} N^{*}(p, q, G) .
\end{aligned}
$$

Собирая оценки, получаем

$$
\sup _{x \in G, x_{n}<l} N(p, q, x)<\infty .
$$

Пусть теперь $x \in G, x_{n} \geqslant l$. Отрезок $\left[0, t_{x}\right]$ разобьем на части $\left[0, \tau_{x}\right],\left[\tau_{x}, t_{x}\right]$, где $\tau_{x}=\min \left\{\rho, t_{x}\right\}$. Тогда

$$
N(p, q, x) \leqslant c N_{0}(p, q, x) \leqslant c\left(N_{1}(p, q, x)+N_{5}(p, q, x)\right)
$$

где $N_{0}$ имеет тот же вид, что и при $x_{n}<l$, а $N_{j}, j=1,5$, отличаются от $N_{0}$ лишш заменой отрезка интегрирования $\left[0, t_{x}\right]$ соответственно на $\left[0, \tau_{x}\right]$ и $\left[\tau_{x}, t_{x}\right]$.

Выражение $N_{1}$ имеет тот же вид, что и при $x_{n}<l$, и оценивается так же, как в (6.17).

Выражение $N_{5}$ оценивается так же, как $N_{2}$ в случае $x_{n}<l$, а именно,

$$
N_{5} \leqslant C_{12} \sup _{0<h \leqslant l}\left(\int_{\tau_{x}}^{t_{x}} \chi\left(\frac{h}{\left(1+2 \delta_{0}\right) t}\right) t^{(s-n / p) p^{\prime}-1} d t\right)^{1 / p^{\prime}} h^{n / q} \leqslant C .
$$

Следовательно,

$$
\sup _{x \in G, x_{n} \geqslant l} N(p, q, x)<\infty .
$$

Из (6.18), (6.19) в силу теорем 6.1, 6.2 получаем утверждения соответственно теорем $6.6,6.7$.

Приведем пример, показывающий, что условие (6.13) является не только достаточным, но и необходимым для справедливости утверждения теоремы 6.5 (при выполнении прочих ее условий). Такого рода пример, относящийся к вложению пространства Соболева $W_{p}^{l}(G) \subset L_{q}(G)$, построен Лабутиным [20] на основе области типа Никодима. Эта же область используется и в нашем случае. 
ПРимеР. Для $n \geqslant 2, \sigma>1$ рассмотрим область $G \subset \mathbb{R}^{n}$, имеющую вид

$$
G=P \cup \bigcup_{j=1}^{\infty}\left(Q_{j} \cup R_{j}\right)
$$

где $P=(0,1)^{n-1} \times(-1,0)$ и

$$
Q_{j}=Q_{j}^{\prime} \times\left(r_{j}, 2 r_{j}\right)=\left(\frac{r_{j}}{2}, \frac{3 r_{j}}{2}\right)^{n-1} \times\left(r_{j}, 2 r_{j}\right)
$$

- куб, а

$$
R_{j}=\left(r_{j}-\frac{r_{j}^{\sigma}}{2}, r_{j}+\frac{r_{j}^{\sigma}}{2}\right)^{n-1} \times\left[0, r_{j}\right]
$$

- полуоткрытый параллелепипед. При этом потребуем, чтобы $0<r_{j} \leqslant 1 / 2, Q_{i}^{\prime} \cap Q_{j}^{\prime}=\varnothing$ при $i \neq j$. Последнее условие вьполняется в случае, например, когда $r_{j+1} \leqslant r_{j} / 4$ при всех $j \in \mathbb{N}$.

Пусть $\eta \in C^{\infty}\left(\mathbb{R}^{1}\right), \eta(t)=0$ при $t \leqslant 1 / 3, \eta(t)=1$ при $t \geqslant 2 / 3$. Положим

$$
f_{j}= \begin{cases}1 & \text { на } Q_{j}, \\ \eta\left(\frac{x_{n}}{r_{j}}\right) & \text { на } R_{j}, \\ 0 & \text { в остальных случаях. }\end{cases}
$$

Тогда [20]

$$
\left\|f_{j} \mid L_{q}(G)\right\|=\left(r_{j}^{n}+r_{j}^{\sigma(n-1)+1}\left\|\eta \mid L_{q}((0,1))\right\|^{q}\right)^{1 / q} \geqslant c r_{j}^{n / q}
$$

где $c$ не зависит от $j$.

Оценим сверху $\Delta^{m}(h, G) f_{j}$ через производные от $f_{j}$. Имеем

$$
\left|\Delta^{m}(h, G) f_{j}(x)\right| \leqslant C|h|^{m} \max _{|y-x| \leqslant|h|} \sum_{|\alpha|=m}\left|D^{\alpha} f_{j}(y)\right| .
$$

Заметим, что при достаточно большом $j\left|\Delta^{m}(h, G) f_{j}(x)\right|$ может отличаться от нуля лишь при $x \in R_{j}$ и при $m|h| \leqslant r_{j}^{\sigma}$. Поскольку при $y \in R_{j},|\alpha|=m$

$$
\left|D^{\alpha} f_{j}(y)\right| \leqslant C_{1} r_{j}^{-m} \eta^{(m)}\left(\frac{y_{n}}{r_{j}}\right) \leqslant C_{1} r_{j}^{-m},
$$

имеем

$$
\left|\Delta^{m}(h, G) f_{j}(x)\right| \leqslant C_{2} \begin{cases}|h|^{m} r_{j}^{-m} & \text { при } m|h| \leqslant r_{j}^{\sigma}, \\ 0 & \text { при } m|h|>r_{j}^{\sigma} .\end{cases}
$$

Следовательно, при $m|h| \leqslant r_{j}^{\sigma}$

$$
\begin{aligned}
\left\|\Delta^{m}(h, G) f_{j} \mid L_{p}\right\|^{p} & \leqslant C 9|h|^{m p} r_{j}^{-m p} r_{j}^{\sigma(n-1)+1}, \\
\left\|f \mid b_{p p}^{s(m)}(G)\right\|^{p} & \leqslant C r_{j}^{-m p+(m-s) \sigma p+\sigma(n-1)+1} .
\end{aligned}
$$

Сравнение последней оценки с (6.20) при $j \rightarrow \infty$ показьвает, что необходимым условием для вложения $B_{p p, r}^{s(m)}(G) \subset L_{q}(G)$, характеризуемого неравенством (6.12), является соотношение (6.13).

Аналогично показывается, что (6.13) необходимо и для вложения (6.11). 
ЗАмЕчАнИЕ 6.1. Из рассмотренного примера следует, в частности, что пространства $B_{p p, r}^{s\left(m^{\prime}\right)}(G), B_{p p, r}^{s\left(m^{\prime \prime}\right)}(G), s<m^{\prime}<m^{\prime \prime}$, отличающиеся лишь использованием разностей различных порядков в их норме, вообще говоря, отличаются друг от друга по запасу элементов (их нормы не эквивалентны).

\section{СПИСОК ЦИТИРОВАННОЙ ЛИТЕРАТУРЫ}

[1] Бесов О.В. Теорема вложения Соболева для области с нерегулярной границей // Докл. РАН. 2000. T. 373. № 2. C. 131-134.

[2] Бесов О.В. Теорема вложения Соболева для области с нерегулярной границей // Матем. сб. 2001. Т. 192. № 3. С. 3-26.

[3] Никольский С. М. Приближение функций многих переменных и теоремы вложения. М.: Наука, 1977.

[4] Бесов О. В., Ильин В. П., Никольский С. М. Интегральные представления функций и теоремы вложения. М.: Наука, 1996.

[5] Трибель Х. Теория интерполяции, функциональные пространства, дифференциальные операторы. М.: Мир, 1980.

[6] Strichartz P.S. Multipliers on fractional Sobolev spaces // J. Math. Mech. 1967. V. 16. № 9. P. 1031-1060.

[7] Лизоркин П. И. Операторы, связанные с дробньм дифференцированием, и классы дифференцируемых функций // Тр. МИАН. 1972. Т. 117. С. 212-243.

[8] Трибель Х. Теория функциональных пространств. М.: Мир, 1986.

[9] Аджиев С.С. Характеризация функциональных пространств $B_{p, q}^{s}(G), L_{p, q}^{s}(G), W_{p}^{s}(G)$ и вложения в $B M O(G) / /$ Тр. МИАН. 1997. Т. 214. С. 7-24.

[10] Triebel H. Local approximation spaces // Z. Anal. Anwend. 1989. V. 88. № 3. P. 261-288.

[11] Брудный Ю. А. Пространства, определяемые с помощью локальных приближений // Тр. MMO. 1971. T. 24. C. 70-132.

[12] DeVore R. A., Sharpley R. S. Maximal function measuring smoothness // Mem. Amer. Math. Soc. 1984. V. 293.

[13] Calderon A.P. Estimates for singular integral operators in terms of maximal functions // Stud. Math. 1972. V. 44. P. 563-682.

[14] Бесов О.В.Эквивалентные нормы в пространствах функций дробной гладкости на произвольной области // Матем. заметки.. Т. 74. № 3. С. 340-349.

[15] Kilpeläinen T., Malý J. Sobolev inequalities on sets with irregular boundaries // Z. Anal. Anwend. 2000. V. 19. № 2. P. 369-380.

[16] Бесов О.В.О компактности вложений весовых пространств Соболева на области с нерегулярной границей // Тр. МИАН. 2001. Т. 232. С. 72-93.

[17] Стейн И. Сингулярные интегралы и дифференциальные свойства функций. М.: Мир, 1973.

[18] Бесов О.В. Вложения пространств дифференцируемых функций переменной гладкости // Тр. МИАН. 1997. Т. 214. С. 25-58.

[19] Бесов О.В.Пространства функций дробной гладкости на нерегулярной области // Докл. PAH. 2002. Т. 383. № 5. С. 586-591.

[20] Лабутин Д. А. Неулучшаемость неравенств Соболева для класса нерегулярных областей // Тр. МИАН. 2001. Т. 232. С. 218-222. 